\title{
Numerical estimation of thermal load in a three blade vertically agitated mixer
}

\author{
Anshul Singh Tomar ${ }^{1}$, Harish $\mathrm{K} \mathrm{G}^{1}$, and $\mathrm{K}$ Arul Prakash ${ }^{1, *}$ \\ ${ }^{1}$ Department of Applied Mechanics, IIT Madras, Chennai, India
}

\begin{abstract}
The objective of this study is to understand the flow physics and resulting heat transfer behind the mixing of highly viscous solid propellant in a vertical three blade mixer. The mixer comprises a four-winged central agitator rotating in the counter-clockwise direction and two other two-winged agitators rotating in clockwise direction. The temperature rises due to the shearing of the solid propellant. Uncontrolled temperature rise may result in the self-ignition of the propellant and other fire hazards. Thus it becomes important to quantify the heat generated due to viscous dissipation to attain a controlled atmosphere for mixing. A detailed CFD analysis is carried out, and two-dimensional energy equation with viscous dissipation term is solved to quantify the temperature rise due to viscous dissipation. The effect of angular velocity of the agitator and viscosity of the propellant over temperature rise is studied quantitatively using the overset method in OpenFOAM. The maximum velocity of the propellant is observed the tip of agitators, whereas maximum temperature rise is found around the vicinity of the blade profile. A correlation is proposed to predict the temperature rise with time due to the viscous effect for the given range of angular velocity.
\end{abstract}

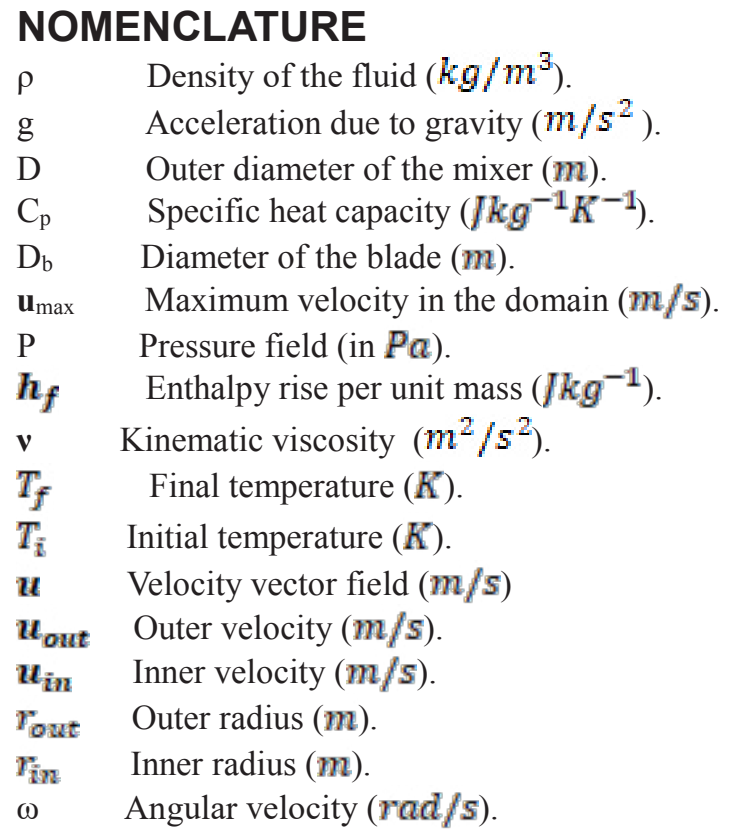

\section{INTRODUCTION}

The mixing process of solid-liquid ingredients in a vertically agitated mixer is a common operation, and it has found application in many industries such as chemical, biological, food industries, etc. One of the most important application of mixers is in the space industry, where solid propellant mixing is carried out to produce rocket fuel. Based on the Reynolds number, the mixing process is categorized into laminar, transition and turbulent mixing. Mixing of highly viscous solid propellant is generally carried out in laminar regime. Complexity while mixing in laminar regime increases due to the formation of isolated mixing regions (IMRs). IMRs are formed in the mixing region due to inefficient mixing, and it requires more power to mix it uniformly [1]. During composite mixing of highly viscous solid propellant with solid ingredients, the temperature rise is observed mainly due to two reasons, the heat of reaction and viscous dissipation. Later becomes predominant while mixing process of solid propellant because, with the increase in viscosity, heat generation due to shearing action also increases. Tanguy et al. [2] emphasized the difficulty in acquiring the required ballistic and mechanical properties from the mixing process in the laminar region since the fluid is paste-like and highly viscous. Debab et al. [3] experimentally studied the effects of baffles, impeller diameter, and the speed of the impeller over the overall heat transfer coefficient, and concluded that the film heat transfer coefficient was strongly dependent on the speed of the impeller. In another flow field visualization experiment Zhang et al. [1] traced the trajectory of flow field particles using digital image processing. The study shows that path line of tracer is continuously disturbed in the opposite direction with another counter rotating path line, which results in less formation of IMRs. Coesnon et al. [4] developed a three-dimensional numerical strategy for simulating viscous fluid flow in complex mixers using a

\footnotetext{
*Corresponding author: arulk@iitm.ac.in
} 
virtual finite element method introduced by Bertrand et al. [5] based upon the fictitious domain method. The kinetic constraints were imposed through Lagrange multipliers on internal blades which were discretized using control points. The mixers used in the present are of two types, horizontal sigma mixers and vertical change-can mixers. For increasing production capacity of these mixers in space industries, the development of such a large scale mixer is carried out for the first time in India. Presently PIV technology and experimental works are carried out to understand the flow physics behind mixing, but these are time-consuming. In this work for the very first-time complex mixing process is simulated using OpenFOAM and viscous heating is approximated.

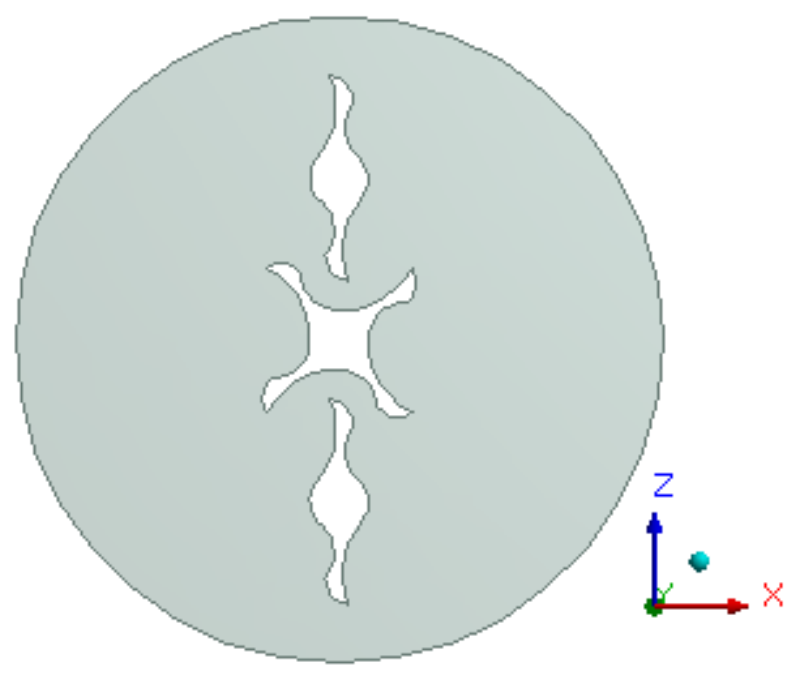

Fig. 1. Computational domain for study

\section{ANALYSIS AND MODELLING}

The governing equations for simulating the incompressible and transient mixing process of highly viscous solid propellant includes solving the NavierStokes equation coupled with energy equation. Mass conservation is represented by Equation 1. Equation 2 shows the conservation of momentum equation where $v \nabla^{2} \boldsymbol{u}$ term represents the momentum transfer due to viscosity, which is dominant in the solid propellant due to its high viscosity.

$$
\begin{gathered}
\frac{\partial \rho}{\partial t}+\nabla \cdot(\rho \boldsymbol{u})=0 \\
\frac{\partial \boldsymbol{u}}{\partial t}+(\boldsymbol{u} \cdot \nabla) \boldsymbol{u}=-\frac{1}{\rho} \nabla p+v \nabla^{2} \boldsymbol{u}
\end{gathered}
$$

The higher viscosity of mixing ingredients promotes the formation of IMRs due to lesser transport of momentum to adjacent particles. Equation 3 describes the conservation of energy.

$$
\frac{\partial T}{\partial t}+v \cdot \nabla T=\frac{k}{\rho C_{p}} \nabla^{2} T+\frac{\left(\mu \phi_{v}\right)}{\rho C_{p}}
$$

$$
\begin{gathered}
\left(\mu \phi_{v}\right)=2 \mu\left[\left(\frac{\partial u_{x}}{\partial x}\right)^{2}+\left(\frac{\partial u_{y}}{\partial y}\right)^{2}-\frac{1}{3}(\nabla \cdot u)^{2}\right] \\
+\mu\left[\frac{\partial u_{y}}{\partial x}+\frac{\partial u_{x}}{\partial y}\right]^{2} \\
\boldsymbol{h}_{f}=C_{p}\left(T_{f}-T_{i}\right)
\end{gathered}
$$

In Equation 3, $\left(\mu \phi_{v}\right)$ term represents the heat interaction taking place due to the effect of shearing while mixing. During the mixing process of highly viscous solid propellant heat generation due to viscous effects dominates the heat of reaction, in this work viscous dissipation term is approximated using finite volume discretization. Equation 5 is used to calculate the enthalpy rise in the process, where $T_{f}$ represents the final temperature rise due to viscous dissipation of heat. Continuity equation along with momentum and energy equation are solved, subjected to the initial and boundary condition as discussed in the next section.

The computational domain, as shown in Figure 1, consists of three agitators of diameter $1.4 \mathrm{~m}$. Two agitators with two vanes are at an offset distance of 0.8 $m$ with the center blade. Agitator with four vanes is rotating at $6.28 \mathrm{rad} / \mathrm{s}$ in the anti-clockwise direction, and other two agitators with two vanes are rotating at $6.28 \mathrm{rad} / \mathrm{s}$ in the clockwise direction. Overset zone conformal to geometry is used. Dynamic viscosity of solid propellant as $450 \mathrm{~Pa} . \mathrm{S}$ is adopted. The temperature and pressure are initialized at $300 \mathrm{~K}$ and 1 bar respectively over the domain. The outer wall of the mixer is subjected to no-slip and agitators are subjected to moving wall velocity boundary conditions. The outer wall and agitator are constrained to zero pressure and temperature gradient.

An open-source $\mathrm{C}++$ toolbox named OpenFOAM for CFD simulations is used. In this work, we choose the PIMPLE algorithm for solving incompressible and transient two dimensional Navier-Stokes equation. PIMPLE is a combination of PISO (Pressure Implicit and Splitting of Operator) and SIMPLE(Semi-Implicit Method For Pressure Linked Equation). PIMPLE solver is used for better stability since the maximum Courant

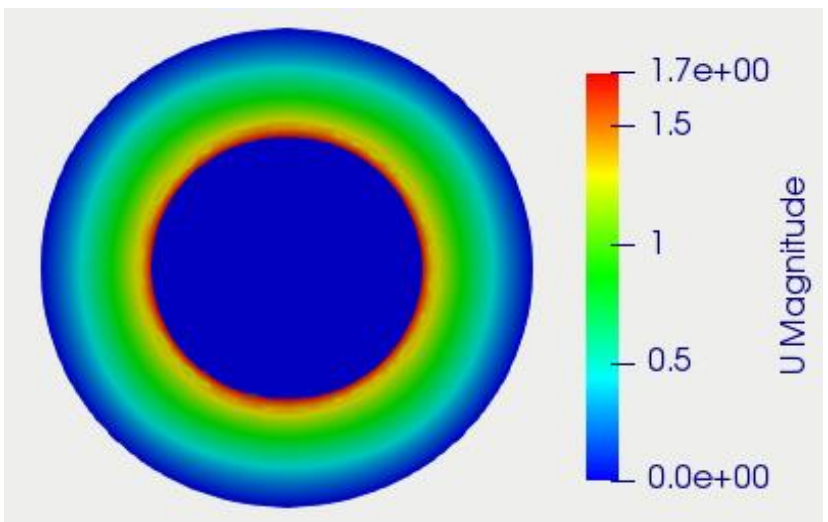

Fig. 2. Taylor-Couette flow using overset method 


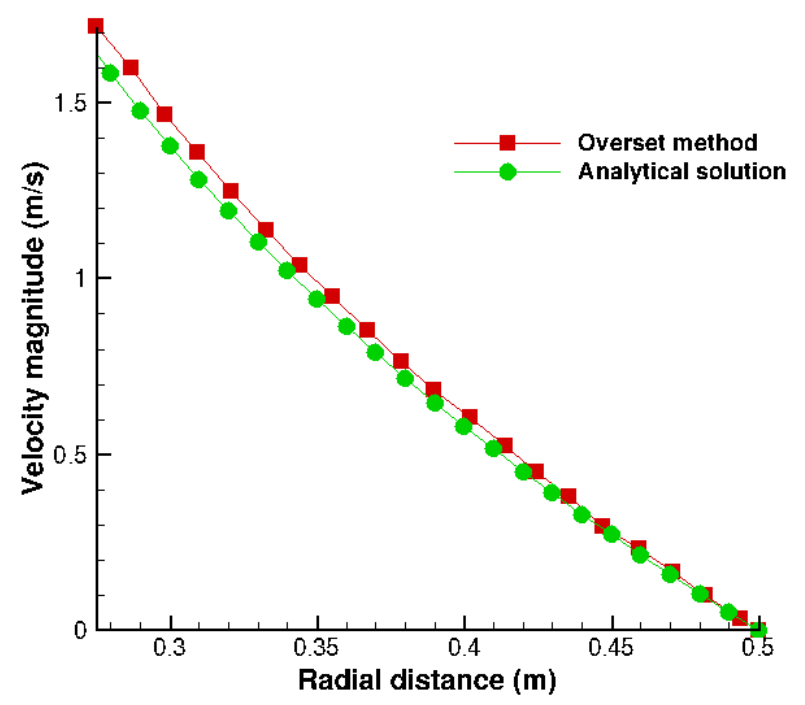

Fig. 3. Comparison of CFD result with analytical solution

Table 1

Grid independence study

\begin{tabular}{lr}
\hline Number of elements & $\mathbf{u}_{\max }(\mathrm{m} / \mathrm{s})$ \\
\hline 83478 & 2.05339 \\
103010 & 2.09372 \\
128693 & 2.08663 \\
\hline
\end{tabular}

number is consistently above 1 . Due to the complex motion of the blade and inter-meshing of vanes overset method is used. For interpolation of data in overset mesh, inverse distance method is used. The gauss-linear scheme is used to solve for the gradients of pressure, velocity and temperature terms. The divergence of velocity is solved by implementing Gauss-linear upwind scheme. In this work, the effect of angular velocity and viscosity over the average temperature rise is studied.

The present solver has been validated using TaylorCouette flow, which consists of two concentric cylinders, as shown in Figure 2. The inner cylinder moves with an angular velocity of $6.28 \mathrm{rad} / \mathrm{s}$, and the outer cylinder is stationary. Analytical solution for Taylor-Couette flow is given by Equation 6 .

$$
\begin{array}{r}
\boldsymbol{u}(\boldsymbol{r})=A r+\frac{B}{r} \\
A=\frac{u_{\text {out }} r_{\text {out }}-u_{\text {in }} r_{\text {in }}}{r_{\text {out }}^{2}-r_{\text {in }}^{2}} \\
B=\boldsymbol{u}_{\text {in }} r_{\text {in }}-A r_{\text {in }}^{2}
\end{array}
$$

Radial velocity profile obtained with overset and the analytical solution is in reasonable agreement, as shown in Figure 3. Grid independence study is carried out as shown in Table 1. Since an increase in the number of elements from $1,03,010$ to $1,28,693$ does not change $\mathbf{u}_{\max }$ significantly, grid corresponding to $1,03,010$ elements is chosen for this study.

\section{RESULT AND DISCUSSION}

To understand the flow physics behind complex mixing process of solid propellant CFD simulations are performed over three agitator system. The velocity and temperature contours are plotted in Figure 4 and Figure 5 , respectively. Uniform wetting of solid propellant is predicted due to minimum clearance between the wing of the outer agitator and central agitator. Critical zones during mixing are identified on contours, and the possible formation of IMRs is predicted in the regions far from the agitators. Temperature rise due to viscous dissipation is quantified, and effect of the temperature rise is predicted within the region covered by the outer agitator. Due to the excessive time taken by the overset method, a correlation is proposed to predict the temperature rise over further time steps.

\section{Velocity and temperature contours}

Velocity contours are obtained with central agitator along with other two agitator rotating at $6.28 \mathrm{rad} / \mathrm{s}$. As shown in Figure 4, the maximum velocity of $2.093 \mathrm{~m} / \mathrm{s}$ is observed near the tip of agitators. Due to the high viscosity of solid propellant very slow advection of velocity is observed towards the mixer wall and momentum transfer in far regions from agitator profile is found to be minimum. Due to less momentum transfer possibility for formation of IMRs increases rapidly. Formation of IMRs represent the improper wetting of solid ingredients and hence for getting better ballistic and mechanical properties from the fuel generated by mixer additional power is required.

In the temperature contour, as shown in Figure 5 temperature rise due to viscous heating is approximated using OpenFOAM. The central agitator is rotated with $6.28 \mathrm{rad} / \mathrm{s}$ in the counter-clockwise direction, and the other two agitators are rotated with $6.28 \mathrm{rad} / \mathrm{s}$ in the clockwise direction. The maximum temperature rise of $0.54 \mathrm{~K}$ with enthalpy rise of $540 \mathrm{Jkg}^{-1}$ is observed for 20 seconds of simulation. Due to the high viscosity of solid propellant and low angular velocity of agitator, diffusion of heat in far regions from agitator is found to be less.

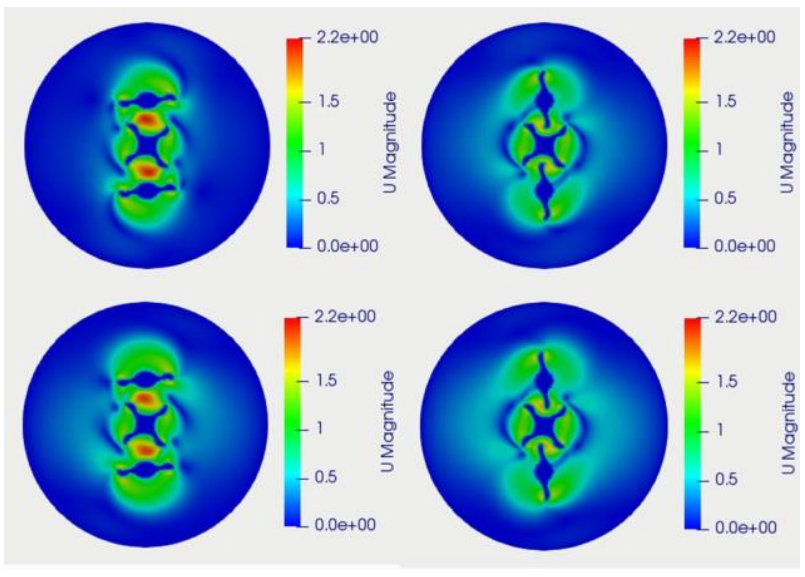

Fig. 4. Velocity contour at time $=0.25,0.5,0.75$ and 1.0 seconds respectively 


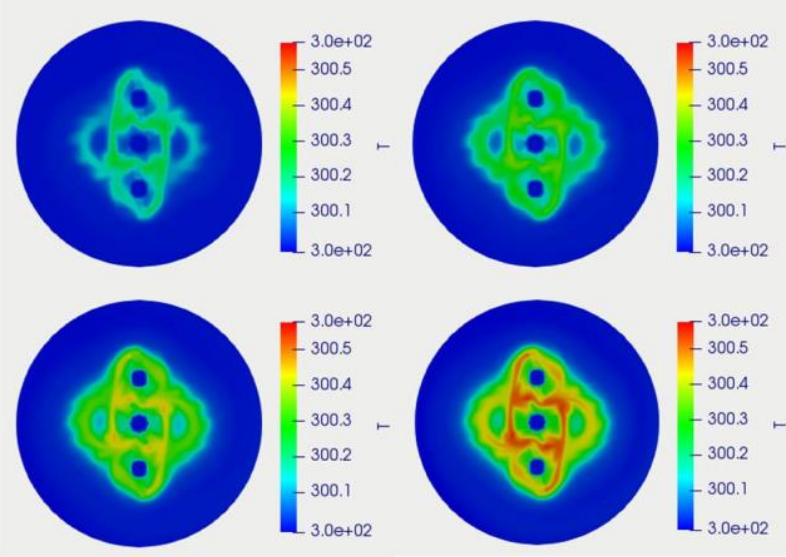

Fig. 5. Temperature contour at time $=5,10,15$ and 20 seconds respectively

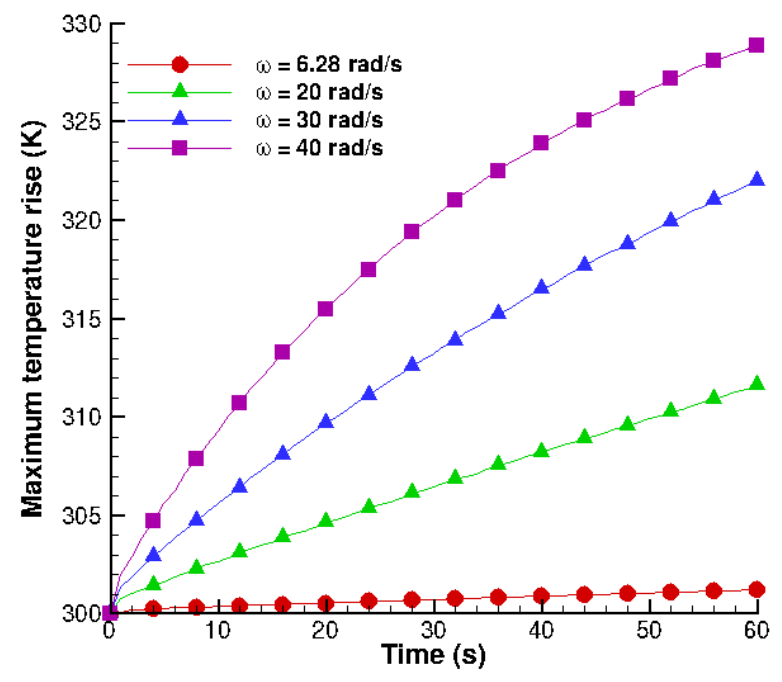

Fig. 6. Effect of angular velocity over temperature rise

\section{Effect of angular velocity and viscosity over maximum temperature rise}

For understanding the effect of angular velocity and viscosity over temperature rise due to viscous heating, CFD simulations are carried out for different angular velocities and viscosity and plots are obtained as shown in Figure 6 and Figure 7. In both Figures, an initial rapid rise in temperature is observed because of the higher temperature gradient between adjacent particles of solid propellant. With time, temperature gradient value decreases and uniform rise in temperature is seen. For increasing mixing efficiency, increase in angular velocity is not viable as temperature rise also increases as can be seen in Figure 6. With an increase in angular velocity, the rate of advection is accelerated which results in increasing the maximum temperature rise as shown in Figure 6. The maximum temperature rise of $28.4 K$ is observed for 60 seconds of simulation with angular velocity of $40 \mathrm{rad} / \mathrm{s}$.

Effect of viscosity over temperature rise is found to be having a lower impact as a maximum temperature rise of $3.4 \mathrm{~K}$ with an enthalpy rise of $3400 \mathrm{Kkg}^{-1}$ is observed for dynamic viscosity of $1050 \mathrm{~Pa} . \mathrm{s}$. With an increase in viscosity of propellant, the power required for mixing increases hence more amount of mechanical energy is converted to heat which increases the temperature of solid propellant as shown in Figure 7.

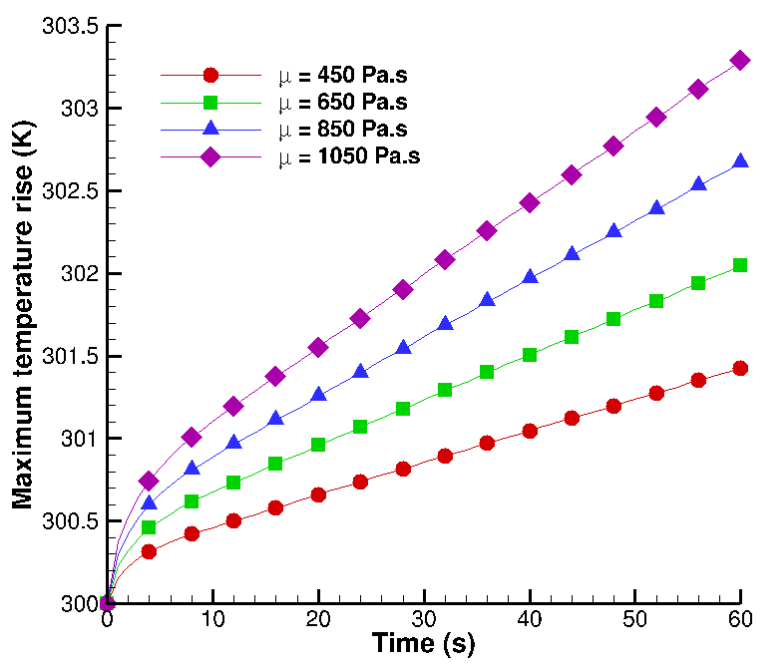

Fig. 7. Effect of viscosity over temperature rise

Simulation run time for getting 60 seconds of simulation with $\Delta \mathrm{t}=0.025 \mathrm{~s}$ was approximately 16 hours. Due to excessive time taken by CFD simulation, a correlation is proposed in Equation 7 using nonlinear regression analysis for predicting the temperature rise due to the shearing effect,

$$
y=a+b x_{1}+c x_{2}+d x_{1}^{2}+e x_{2}^{2}+f x_{1} x_{2}
$$

Table 2

Variable value with $99 \%$ confidence level

\begin{tabular}{cc}
\hline Variable & Value \\
\hline $\mathrm{a}$ & 299.159 \\
$\mathrm{~b}$ & 0.0604 \\
$\mathrm{c}$ & -0.0738 \\
$\mathrm{~d}$ & -0.00207 \\
$\mathrm{e}$ & 0.0047 \\
$\mathrm{f}$ & 0.0129 \\
\hline
\end{tabular}

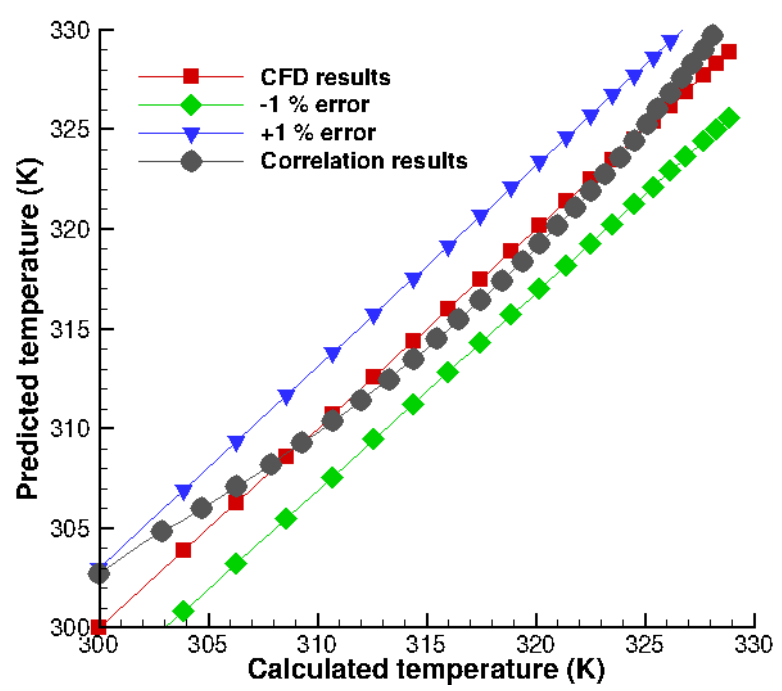

Fig. 8. Comparison of CFD results with correlation within \pm 1 $\%$ error 
Where $x_{1}$ refers to the time $(s), x_{2}$ refers to angular velocity $(\mathrm{rad} / \mathrm{s})$ and $\mathrm{y}$ refers to the predicted temperature rise (K). Variable values with a 99\% confidence level are tabulated in Table 2. Equation $7 \mathrm{can}$ be used to predict the temperature rise for $6.28<\omega<40$. Predicted temperature rise is plotted and compared against the actual results from CFD and predicted temperature rise is bounded within $\pm 1 \%$ error, as shown in Figure 8.

\section{CONCLUSIONS}

In this work mixing process of highly viscous solid propellant is simulated using CFD technique in OpenFOAM. Maximum velocity of $2.093 \mathrm{~m} / \mathrm{s}$ is observed near the tip of the agitator. The maximum temperature rise due to viscous heating is approximated to be $0.54 K$ for 20 seconds of mixing. Momentum transfer due to advection is found to be minimum in the regions far from blade profile owing to the high viscosity of solid propellant hence temperature rise is also maximum in the vicinity of agitator profile. A correlation is proposed to predict the temperature rise within bound of $\pm 1 \%$ error for given angular velocity and time. It may be concluded that with increase in angular velocity mixing can be improved, but it results in significant temperature rise hence with lower angular velocity use of additional motion for agitators is suggested for better mixing.

The authors thank Indian Space Research Organisation for funding and $\operatorname{Dr} \mathrm{T}$. Jayachandran (visiting professor, Department of Aerospace engineering, IIT Madras) for his technical support.

\section{REFERENCES}

1. J Zhang, X Li, R. H., and Liang, J., Adv. in Mech. Engg., 7(7), 1-12 (2015)

2. PA Tanguy, F Thibault, D. C., and A, Chem. Engg. Res. and des., A.-K., 318-324 (1999).

3. A. Debab, N. Chergui, K. B., and J. Bertrand, 2011. J. of app. fluid mech., 4, 43-50 (2011).

4. B. Coesnon, M. Heniche, C. D. F. B., and P.A. Tanguy. Intl. J. for num. methd. in fluids, 427-449 (2008).

5. F. Bertrand, T. P., and F, T. Intl. J. for num. methd. in fluids, 719-736 (1997). 